\title{
El bienestar animal como eje de la comunicación digital del sector alimentación: los subsectores cárnico y lácteo
}

\section{Animal welfare as a central theme of digital communication in the food sector: meat and dairy subsectors}

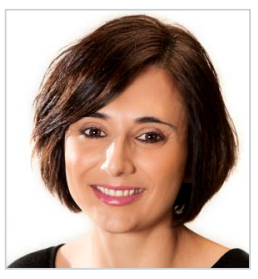

\begin{abstract}
Araceli Castelló-Martínez. Doctora en Comunicación por la Universidad Cardenal Herrera CEU, Máster en Comunicación Integral por la Universidad Complutense de Madrid, Máster Ejecutivo en Community Management por la Universidad de Alicante y Licenciada en Publicidad y RR.PP. por la Universidad de Alicante, con premio extraordinario de Licenciatura y segundo premio nacional. Acreditada como profesora Titular de Universidad por ANECA y con un sexenio por parte de la AVAP (2009-2015) y de CNEAI (2011-2017), imparte docencia en los estudios de Publicidad y RR.PP. en la Universidad de Alicante desde 2009. Ha sido también profesora en la Licenciatura en Publicidad y RR.PP. de la Universidad CEU Cardenal Herrera (2008-2010) y ha colaborado como profesora en diversos postgrados. Ha trabajado en agencias de medios (Netthink, Initiative)y soportes digitales(Ya.com, Vocento) en Españay Bélgicay realizado numerosas publicaciones sobre comunicación digital, como los libros Estrategias empresariales en la Web 2.0. Las redes sociales online (2010), La comunicación en cambio constante (2013) o De la publicidad a la comunicación persuasiva integrada. Estrategia y empatía (2019).

Universidad de Alicante, España

araceli.castello@ua.es

ORCID: 0000-0001-5783-344X
\end{abstract}

Recibido: 16/09/2020 - Aceptado: 18/05/2021 - En edición: 03/09/2021 - Publicado: 01/12/2021

\section{Resumen:}

El consumidor actual, cada vez más preocupado por practicar una alimentación saludable y de calidad, demanda transparencia a las empresas de alimentación y les exige que respeten las condiciones en las que se crían los animales, concepto conocido como bienestar animal. Certificaciones como la de AENOR acreditan la buena alimentación, el buen alojamiento, la buena salud y el comportamiento apropiado del animal. El principal objetivo del estudio es analizar la comunicación del bienestar animal en espacios digitales como la página web y las redes sociales por parte de las principales marcas españolas de los subsectores de alimentación cárnico y lácteo. A partir de cuatro dimensiones -certificación, difusión, audiencia e interacción y estrategia creativa-, se realiza un análisis cuanti/cualitativo de las publicaciones realizadas por 21 marcas durante 2019 y el primer semestre de 2020. Los resultados avalan la presencia del bienestar animal como eje de comunicación en las estrategias creativas de la comunicación digital de las marcas de los subsectores cárnico y lácteo. Estos mensajes híbridos fusionan su intencionalidad comercial con el compromiso de la marca por dar respuesta a una tensión social y cultural y fomentan la interacción y la participación del usuario, característica intrínseca a la comunicación digital.

\section{Palabras clave:}

Bienestar animal; redes sociales; estrategia creativa; comunicación digital; alimentación.

\footnotetext{
Cómo citar este artículo:

Castelló-Martínez, A. (2021). El bienestar animal como eje de la comunicación digital del sector alimentación: los subsectores cárnico y lácteo. Doxa Comunicación, 33, pp. 179-196.
}

\section{Abstract:}

Today's consumer, increasingly concerned about healthy and quality eating, demands transparency from food companies and demands that they respect the conditions in which animals are raised, a concept known as animal welfare. Certifications such as AENOR credit good food, good accommodation, good health and proper behavior of the animal. The main goal of the study is to analyze the communication of animal welfare in digital spaces such as the website and social networks by the main Spanish brands ofmeat and dairy food subsectors. From four dimensions -certification, dissemination, audience and interaction and creative strategy-, a quantitative/qualitative analysis of publications by 21 brands during 2019 and the first half of 2020 is carried out. Results support the presence of animal welfare as communication hub in the creative strategies of digital communication of brands in meat and dairy subsectors. These hybrid messages fuse their commercial intentionality with the brand's commitment to respond to a social and cultural tension and encourage user interaction and participation, a characteristic intrinsic to digital communication.

\section{Keywords:}

Animal welfare; social networks; creative strategy; digital communication; food. 


\section{Introducción}

\subsection{La preocupación por lo que comemos, una tendencia del consumidor creciente}

Con la intensificación de los sistemas de producción animal se ha acrecentado también la preocupación de la sociedad por los efectos que ésta tiene en el bienestar de los animales (Alonso, González-Montaña y Lomillos, 2020): el porcentaje de ciudadanos europeos que considera muy importante proteger el bienestar de los animales de granja creció del 34\% al 57\% entre 2006 y 2015. De acuerdo con este estudio, los productos "amigables con los animales" son percibidos como más saludables y de mayor calidad y cada vez son más los consumidores que se niegan a comprar productos que no respeten el bienestar animal.

Esta conciencia se enmarca en la cultura activista que ha traído consigo la participación ciudadana en las redes sociales: "los usuarios elevan su voz denunciando aquello que no es coherente, verdadero, natural, transparente o auténtico" (Castelló-Martínez y Del Pino-Romero, 2019: 167). El consumidor está más comprometido con su entorno, se preocupa por temas ambientales o ecológicos y busca un modelo de vida más limpio, sano y respetuoso consigo mismo, con los demás y con el mundo que les rodea. La búsqueda de experiencias sanas hace que en la alimentación crezca la demanda por lo orgánico, lo natural y lo sostenible. La idea de que "somos lo que comemos" ya no sólo se refiere al impacto de la alimentación en la salud física, sino también en la mental.

La reducción en el consumo de carne se debe, según manifiesta el 22\% de los usuarios, a temas relacionados con el bienestar animal (AECOC, 2020). Frente al consumidor que ama la carne por la ilusión y el disfrute en la experiencia de consumo, el connsumidor que rechaza la carne lo hace por la presión social de que comer carne es malo para la salud y el medio ambiente.

Los consumidores entienden que las condiciones en las que se crían los animales, se manejan los cultivos y se aprovechan los recursos y el trato justo de los animales y los trabajadores que participan en los procesos de producción tienen un impacto directo en la calidad del producto y son determinantes para alcanzar un estilo de vida sano y saludable. El concepto de bienestar animal se asocia con el buen estado de salud de los animales (20\%), la ausencia de dolor en la matanza (17\%), el pasto en libertad (15\%), el cuidado por parte de profesionales veterinarios (13\%), la existencia de espacio suficiente para las necesidades de los animales (13\%) o una buena alimentación (8\%) (AECOC, 2020).

Según la Organización Mundial de Sanidad Animal (OIE)², bienestar es el modo en el que un animal se enfrenta a las condiciones en las que vive. Se entiende que el bienestar es una propiedad inherente de un animal y que incluye los estados físicos y mentales del mismo y, en algunos casos, el grado en que se satisface su naturaleza particular -rasgos genéticos, manifestados como raza y temperamento- (Hewson, 2005).

El consumidor exige transparencia en los métodos de producción de los alimentos y en los procesos de crianza de los animales, para asegurar que no han sufrido maltrato y que han sido criados en unas condiciones dignas: el $94 \%$ de los europeos cree importante que los animales de granja tengan buena calidad de vida y el $37 \%$ está dispuesto a pagar un $5 \%$ más por productos que proceden de sistemas de producción respetuosos con el bienestar animal (Comisión Europea, 2019). El 84\% de los españoles

1 http://www.oie.int 
piensa que es necesario mejorar la protección de los animales en las explotaciones y el 71\% declara que le gustaría tener más información sobre las condiciones en las que viven los animales de granja (Comisión Europea, 2015).

\subsection{El sector alimentación en España y los subsectores cárnico y lácteo}

La industria de alimentación y bebidas es uno de los sectores más importantes en la producción, el empleo y el comercio exterior de España (FIAB, 2020). La cifra de negocio de este sector superó en 2019 los 120.946 millones de euros, representando el 14,9\% del total de la industria manufacturera española (FIAB, 2020). Se trata de un sector en el que la inversión en I+D+i se centra en la elaboración de productos artesanales, biológicos, relacionados con la salud, enriquecidos o producidos con una menor cantidad de aditivos, azúcares, grasas y conservantes, para dar respuesta a la mayor preocupación de un consumidor concienciado y comprometido con el cuidado del medio ambiente, el bienestar animal y la práctica de una alimentación sana, como hemos comentado en el apartado anterior.

El gasto total en alimentación y bebidas ascendió a 105.465,2 millones de euros en 2019 en España (Ministerio de Agricultura, Pesca y Alimentación, 2020). Cada español gastó una media de 2.567,17 euros en alimentación. La carne representa el 20,6\% del consumo doméstico, seguida de las frutas y hortalizas (17,2\%), la pesca (13\%) y la leche y los derivados lácteos (11,3\%). En 2020 el sector alimentación invirtió 293,6 millones de euros en publicidad en España (InfoAdex, 2021), siendo el quinto sector por volumen de inversión. Nestlé, Danone, Mondelez y Ferrero Ibérica son las empresas que ocupan las primeras posiciones en inversión publicitaria.

Alimentación es el sector con mayor presión publicitaria en televisión (Barlovento Comunicación, 2021), con una cuota del 19\%, y representa el 3,3\% de las interacciones que se producen en redes sociales (IAB Spain, 2020), con más de 43,5 millones de seguidores. Tras Coca-Cola, las marcas de gran consumo preferidas por los españoles son El Pozo, Campofrío y Central Lechera Asturiana (Kantar Worldpanel, 2020), todas del sector alimentación y bebidas. El Pozo es la marca que en más hogares entra, con una cuota del $76,8 \%$.

Las redes sociales se han configurado como un canal fundamental en el marketing digital de las marcas del sector alimentación (Youssef, 2019). Un ejemplo de ello es la marca Campofrío Food Group, que ha cosechado un gran éxito con sus campañas publicitarias, en particular a través de plataformas como Twitter (Raya, 2017). El eje de comunicación de las estrategias publicitarias de esta marca dejó de basarse en factores tradicionales o relacionados con la salud y el bienestar físico, para centrarse en la positividad y en la necesidad de tener un buen estado de ánimo, con un tono humorístico, emocional y empático.

La publicidad de las marcas del sector alimentación contribuye a la construcción del concepto de alimentación saludable, frente a la inconsistencia de las recomendaciones alimentarias institucionales (López-Briones, 2017). La publicidad de productos de alimentación no con poca frecuencia recurre a argumentos médico-científicos para promocionar alimentos que se presentan no sólo como saludables, sino también como necesarios para alcanzar el canon de belleza establecido (Rey, 2010) o con virtudes preventivas o curativas (Díaz-Méndez y González-Álvarez, 2013).

Los subsectores cárnico y lácteo representan el 12,1\% y 5,7\% de las empresas de alimentación (FIAB, 2020), respectivamente. El tipo de carne con mayor producción en España es la de porcino $(63,8 \%)$, seguido del de ave (23,9\%). Este subsector cuenta con unas 3000 empresas, la mayoría de ellas pequeñas y medianas. Campofrío Food Group, Bonàrea, El Pozo, Jorge S.L. y Coren son 
las empresas con mayor participación en este subsector, superando cada una de ellas los mil millones de euros en ventas (FIAB, 2020).

Como datos destacables en la demanda, en los hogares donde compra una persona con más de 65 años el consumo de carne es más elevado, mientras que la demanda más reducida se asocia a los hogares donde la compra la realiza una persona con menos de 35 años. Los consumidores que residen en grandes núcleos urbanos -de 100.000 a 500.000 habitantes- consumen menos carne, así como las parejas jóvenes sin hijos, los hogares monoparentales y los jóvenes independientes. Pese a que el cárnico es el principal subsector en alimentación, desde 2014 tanto el consumo como el gasto siguen una tendencia a la baja (FIAB, 2020).

Con respecto al subsector de leche y derivados lácteos, por persona se cuantifica un consumo de 69,8 litros de leche y de 35,5 kilos de productos lácteos al año (FIAB, 2020). La leche es una de las principales producciones ganaderas de España y su importancia económica sólo está por detrás del porcino y el vacuno. Aproximadamente el $88 \%$ de la producción nacional de leche corresponde a la producción de leche de vaca, el 6,2\% a la producción de leche de oveja y el 5,8\% a la producción de leche de cabra (FIAB, 2020). Grupo Lactalis Ibérica, Danone, Capsa y Calidad Pascual son las principales empresas del sector de lácteos.

Los hogares sin niños registran los consumos más bajos y, de nuevo, en los hogares donde compra una persona con más de 65 años, el consumo de leche es más elevado, mientras que la demanda más reducida se asocia a los hogares donde la compra la realiza una persona que tiene menos de 35 años. Desde 2014 el consumo y el gasto en leche también siguen una tendencia decreciente. Además, el precio de la leche ha descendido, lo cual ha generado quejas entre los ganaderos por seguir cobrando lo mismo o menos, pese a la inversión en reformas para adaptar las instalaciones (Valero, 2018). En la familia de leches, la única que crece en demanda es la leche enriquecida.

\subsection{Legislación y certificación del bienestar animal}

En España las primeras normativas para la protección animal datan de 1883, cuando una Real Orden estableció que los docentes debían inculcar entre el alumnado sentimientos de benevolencia hacia los animales. Desde la promulgación del Decreto 2715/78, la protección animal es responsabilidad del Ministerio de Agricultura. La Ley 32/2007 regula el cuidado de los animales, su explotación, transporte, experimentación y sacrificio. El Real Decreto 441/2001 incluye la normativa general básica sobre el bienestar de los animales en las granjas y es la trasposición al ordenamiento jurídico español de la Directiva 98/58/CE. Existen también normativas específicas, como el Real Decreto 692/2010 para la protección de terneros en las explotaciones ganaderas, el Real Decreto 1221/2009, que incluye las normas básicas para las explotaciones de ganado porcino, o el Real Decreto 3/2002 para la protección de las gallinas ponedoras.

La estrategia de la Unión Europea 2012-2015 sobre bienestar animal² tenía como objetivos proporcionar información clara y precisa a los consumidores, simplificar la legislación, mejorar la formación de ganaderos y veterinarios y ayudar a cumplir la legislación ${ }^{3}$. A nivel internacional, la OIE recoge en el Código Sanitario para Animales Terrestres ${ }^{4}$ directrices sobre el sacrificio de animales y la matanza.

\footnotetext{
Puede consultarse en http://bit.ly/ce-animalwelfare

La información sobre cuestiones de bienestar animal de la Unión Europea se encuentra disponible en http://bit.ly/ce-animalwelfare-2

http://bit.ly/oie-bienestaranimal
} 
Ante la necesidad de asegurar una gestión correcta en los sistemas de cría de animales destinados a la producción alimentaria y así hacérselo saber a los consumidores, la Asociación Española de Normalización y Certificación (AENOR) ha creado certificaciones al respecto. Siguiendo el Protocolo Welfare Quality y AWIN (Animal Welfare Indicators), el Instituto de Investigación y Tecnología Agroalimentaria (IRTA) y AENOR crearon en 2014 el certificado AENOR de Bienestar Animal Welfair ${ }^{5}$, que sigue los cuatro pilares de los estándares europeos: buena alimentación, buen alojamiento, buena salud y comportamiento apropiado del animal.

Estos principios se desglosan en 12 criterios: ausencia de hambre prolongada, ausencia de sed prolongada, confort durante el descanso, confort térmico, facilidad de movimiento, ausencia de lesiones, ausencia de enfermedades, ausencia de dolor causado por el manejo, expresión de la conducta social, expresión de otras conductas, buena relación hombre-animal y estado emocional positivo.

En la figura 1 pueden verse estos principios, inspirados en las cinco libertades que Brambell formuló en la década de 1960: libre de hambre y de sed, libre de miedos y angustias, libre de incomodidades físicas o térmicas, libre de dolor, lesiones o enfermedades y libre para expresar las pautas propias de comportamiento. AENOR cuenta también con la certificación privada Leche de Pastoreo.

Figura 1. Principios del Protocolo Welfare Quality

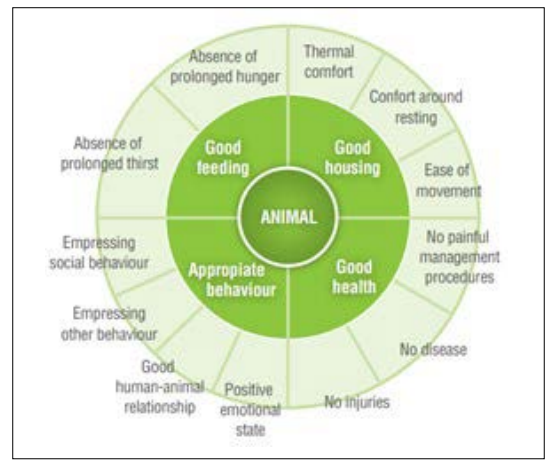

Fuente: www.welfarequality.net

Existen otros sellos concretos para determinadas especies, como el de Interporc Animal Welfare Spain (IAWS) creado por la Organización Interprofesional Agroalimentaria del Porcino de Capa Blanca (Interporc) o el de la Asociación Nacional para la Defensa de los Animales (ANDA) para huevos de gallinas camperas y ecológicas -sus pautas incluyen medidas medioambientales y la apuesta por el desarrollo rural-. El sector cárnico español ha creado también el sello Compromiso Bienestar Certificado para todas las producciones ganaderas. Encontramos también otros certificados específicos para mataderos expedidos por diversas entidades privadas.

5 http://bit.ly/aenor-bienestaranimal 
El Índice de Referencia Empresarial Mundial sobre el Bienestar de los Animales de Granja (BBFAW, 2020) muestra las empresas alimentarias más respetuosas con el bienestar animal y destaca cómo cada vez son más las que realizan inversiones y esfuerzos para adaptar sus sistemas de producción a las certificaciones de bienestar animal. Según el Índice de Protección Animal (API) de World Animal Protection ${ }^{6}$, España se encuentra entre los 50 países que necesita implementar mejoras urgentes relativas al bienestar de los animales, especialmente en aspectos como la tauromaquia y algunas prácticas en la ganadería intensiva como el confinamiento de animales de granja y las jaulas de paridera en la cría de cerdos y en gallinas ponedoras.

\subsection{El bienestar animal en las estrategias comunicativas}

En respuesta a la creciente preocupación del consumidor por el bienestar animal, en los últimos años marcas de los sectores distribución y alimentación han incluido en sus estrategias comunicativas esta cuestión como eje de comunicación o ventaja diferencial. En la figura 2 puede verse la identidad visual de la certificación de AENOR, que las marcas incluyen en el envase de sus productos:

Figura 2. Certificación de bienestar animal de AENOR

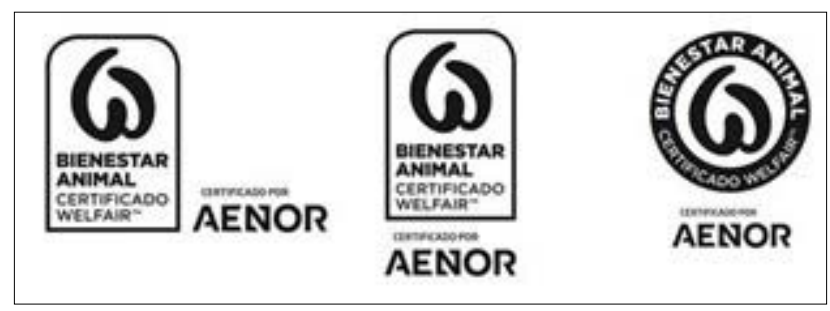

Fuente: http://www.aenor.com

Braunsberger (2015) demostró que las campañas publicitarias en defensa del bienestar animal y de los animales frente a amenazas como la caza obtienen un elevado nivel de influencia en la ciudadanía. En sus acciones de comunicación, las organizaciones que defienden el bienestar animal utilizan para promover cambios de actitud y comportamiento en la audiencia recursos como las imágenes impactantes sobre el sufrimiento de los animales, las pruebas de la inteligencia animal y su socialización o la presencia de celebridades (Sullivan y Longnecker, 2010). La comunicación del bienestar animal también apuesta por exhibir desde un enfoque positivo animales que disfrutan de experiencias placenteras o están "libres de experiencias negativas" (Vigors, 2019).

En España existen diversas cadenas de distribución que incluyen en su discurso publicitario la difusión de las medidas adoptadas en materia de bienestar animal, como Eroski o Lidl. La segunda obtuvo la certificación para la producción de huevos de gallinas criadas en el suelo, convirtiéndose en la primera cadena de distribución en España en garantizar que el 100\% de los huevos que comercializa procede de gallinas criadas en libertad (El Mundo, 2018). Además, cuenta con leche fresca con doble certificación en bienestar animal y pastoreo, el certificado de bienestar animal para su marca de leche Milbona, 100\% nacional, y

6 https://api.worldanimalprotection.org/\# 
el certificado para el pollo fresco. Así lo difunde en sus supermercados y en su página web ${ }^{7}$. En la figura 3 pueden verse ejemplos de algunas de las acciones comunicativas realizadas por Lidl. Eroski publica en YouTube vídeos ${ }^{8}$ en los que los ganaderos con los que trabajan narran cómo cuidan de los animales y muestran las instalaciones de sus granjas.

Figura 3. Acciones de comunicación de Lidl sobre bienestar animal

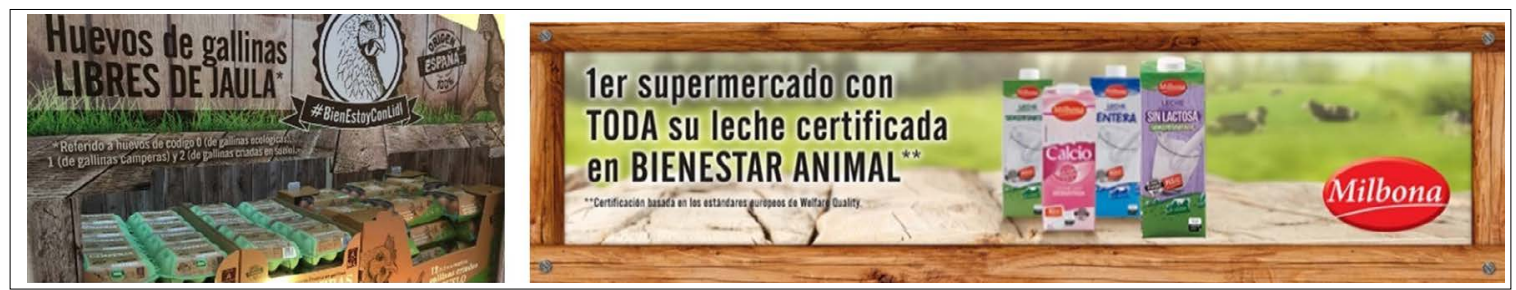

Fuente: Google (www.google.es) y YouTube (www.youtube.com)

En el sector lácteo destacan las estrategias comunicativas de las marcas Leche Pascual y Central Lechera Asturiana. De la mano de la agencia Oriol Villar, en 2019 Leche Pascual lanzaba una campaña en la que rompía con la estrategia tradicional de la publicidad del sector, centrándose en el descanso de las vacas. Spots como "Buenas noches"9 o "Canción" ${ }^{10}$ comunicaban que todas las granjas con las que trabaja la marca tienen el Certificado de Bienestar Animal, bajo el lema de que cuanto mejor esté la vaca, mejor será la leche.

La campaña "Pongámonos de acuerdo"11 (2019), de Ogilvy\&Mather para Central Lechera Asturiana, reivindica los valores de la sostenibilidad y el bienestar animal e invita a reflexionar sobre lo positivo de la colaboración. La misma agencia firma la acción "Lo que aprendimos en casa"12 (2019), que muestra el compromiso de la familia ganadera que es la marca.

\section{Material y métodos}

El principal objetivo del estudio es analizar la comunicación del bienestar animal en espacios digitales como la página web y las redes sociales por parte de las principales marcas españolas de los subsectores cárnico y lácteo. Las preguntas de investigación son las siguientes:

RQ1. ¿Las principales empresas de los sectores cárnico y lácteo cuentan con la certificación de bienestar animal? ¿Se informa en la web de las marcas de dicha certificación?

\footnotetext{
http://bit.ly/lidl-bienestaranimal

Puede verse un ejemplo en http://bit.ly/eroski-yt

https://bit.ly/buenasnoches-leche

https://bit.ly/cancion-lechepascual

http://bit.ly/cla-pongamonos

http://bit.ly/cla-loqueaprendimos
} 
RQ2. ¿Se realizan en redes sociales publicaciones relacionadas con el sello de bienestar animal? ¿Qué porcentaje representan estas publicaciones con respecto al total de publicaciones realizadas?

RQ3. ¿Qué audiencia e interacción reciben las publicaciones en redes sociales de marcas de los sectores cárnico y lácteo sobre el sello de bienestar animal?

RQ4. ¿Existen características comunes en las publicaciones sobre el sello de bienestar animal de las marcas de los sectores cárnico y lácteo en redes sociales?

El trabajo de campo se basa en un análisis cuanti/cualitativo de los contenidos de las páginas web de 21 marcas de los subsectores cárnico y lácteo, pertenecientes a las principales empresas españolas de acuerdo con el volumen de ventas (FIAB, 2020). Se analizan también las publicaciones relativas al bienestar animal y a su certificación realizadas por estas marcas durante el año 2019 y el primer semestre de 2020 en las tres redes sociales con más usuarios en España -Facebook, YouTube e Instagram, tras WhatsApp, de acuerdo con IAB Spain (2021)-. Los datos se recopilaron en julio de 2020. En la tabla 1 se muestra la matriz de congruencia del estudio:

Tabla 1. Matriz de congruencia

\begin{tabular}{|c|c|c|c|}
\hline Dimensión & Cuestión (RQ) & Indicador & Referencia \\
\hline Certificación & $\begin{array}{l}\text { RQ1. ¿Las principales empresas de los sectores cárnico y lácteo } \\
\text { cuentan con la certificación de bienestar animal? ¿Se informa en la } \\
\text { web de las principales marcas de dicha certificación? }\end{array}$ & $\begin{array}{l}\text { 1. Existencia de certificación } \\
\text { 2. Presencia en la web }\end{array}$ & Google, página web \\
\hline Difusión & $\begin{array}{l}\text { RQ2. ¿Se realizan en redes sociales publicaciones relacionadas con } \\
\text { el sello de bienestar animal? ¿Qué porcentaje representan estas } \\
\text { publicaciones con respecto al total de publicaciones realizadas? }\end{array}$ & $\begin{array}{l}\text { 1. Publicaciones sobre } \\
\text { bienestar animal vs } \\
\text { publicaciones totales }\end{array}$ & \multirow{3}{*}{$\begin{array}{l}\text { Facebook, } \\
\text { Instagram, YouTube } \\
\text { Costa-Sánchez, } \\
2017\end{array}$} \\
\hline $\begin{array}{l}\text { Audiencia e } \\
\text { interacción }\end{array}$ & $\begin{array}{l}\text { RQ3. ¿Qué audiencia e interacción reciben las publicaciones en } \\
\text { redes sociales de marcas de los sectores cárnico y lácteo sobre el } \\
\text { sello de bienestar animal? }\end{array}$ & $\begin{array}{l}\text { 1. Visualizaciones } \\
\text { 2. Me gusta, no me gusta, } \\
\text { comentarios }\end{array}$ & \\
\hline $\begin{array}{l}\text { Estrategia } \\
\text { creativa }\end{array}$ & $\begin{array}{l}\text { RQ4. ¿Existen características comunes en las publicaciones sobre } \\
\text { el sello de bienestar animal de las marcas de los sectores cárnico y } \\
\text { lácteo en redes sociales? }\end{array}$ & $\begin{array}{l}\text { 1. Periodicidad en la } \\
\text { publicación } \\
2 \text {. Tipo de publicación } \\
\text { 3. Inclusión de texto, etiquetas } \\
\text { y URL } \\
\text { 4. Tipo de vídeo } \\
\text { 5. Llamada a la acción } \\
\text { 6. Sorteos, concursos, } \\
\text { promociones }\end{array}$ & \\
\hline
\end{tabular}

Fuente: elaboración propia

La tabla 2 incluye la muestra y los 51 canales analizados para las 21 marcas seleccionadas. Como vemos, 20 de las 21 empresas tienen algún espacio propio en las principales redes sociales (95,2\%). Facebook es la plataforma más popular ( $\mathrm{n}=20$; 95,2\%), seguida de Instagram $(\mathrm{n}=16 ; 76,2 \%)$ y de YouTube $(\mathrm{n}=15 ; 71,4 \%)$. De las 21 marcas, $15(71,4 \%)$ tienen presencia en las tres redes sociales. 
Tabla 2. Canales analizados en Facebook, YouTube e Instagram

\begin{tabular}{|c|c|c|c|c|c|}
\hline Empresa & Sector & Marca & Facebook & YouTube & Instagram \\
\hline Calidad Pascual & Lácteo & Pascual & http://bit.ly/pascual-fb & http://bit.ly/pascual-yt & http://bit.ly/pascual-ig \\
\hline Campofrío Food Group & Cárnico & Campofrío & http://bit.ly/campofrio-fb & http://bit.ly/campofrio-yt & $\begin{array}{l}\text { http://bit.ly/campofrio- } \\
\text { ig }\end{array}$ \\
\hline Casa Tarradellas & Cárnico & Casa Tarradellas & $\begin{array}{l}\text { http://bit.ly/ } \\
\text { casatarradellas-fb }\end{array}$ & http://bit.ly/casatarradellas-yt & $\begin{array}{l}\text { http://bit.ly/ } \\
\text { casatarradellas-ig }\end{array}$ \\
\hline $\begin{array}{l}\text { Coop. Ganad. del Valle } \\
\text { de los Pedroches }\end{array}$ & Lácteo & Lácteos COVAP & $\begin{array}{l}\text { http://bit.ly/lacteosCOVAP- } \\
\text { fb }\end{array}$ & http://bit.ly/lacteosCOVAP-yt & $\begin{array}{l}\text { http://bit.ly/ } \\
\text { lacteosCOVAP-ig }\end{array}$ \\
\hline $\begin{array}{l}\text { Coop. Ganad. del Valle } \\
\text { de los Pedroches }\end{array}$ & Lácteo & Ibéricos COVAP & $\begin{array}{l}\text { http://bit.ly/ } \\
\text { ibericosCOVAP-fb }\end{array}$ & http://bit.ly/ibericosCOVAP-yt & $\begin{array}{l}\text { http://bit.ly/ } \\
\text { ibericosCOVAP-ig }\end{array}$ \\
\hline $\begin{array}{l}\text { Cooperativas } \\
\text { Orensanas }\end{array}$ & Cárnico & Coren Grill & http://bit.ly/corengrill-fb & http://bit.ly/corengrill-yt & http://bit.ly/corengrill-ig \\
\hline $\begin{array}{l}\text { Corporación } \\
\text { Alimentaria Guissona }\end{array}$ & Cárnico & Bonàrea Agrupa & http://bit.ly/bonarea-fb & http://bit.ly/bonarea-yt & http://bit.ly/bonarea-ig \\
\hline $\begin{array}{l}\text { Corp. Alim. Peñasanta } \\
\text { (CAPSA Food) }\end{array}$ & Lácteo & $\begin{array}{l}\text { Central Lechera } \\
\text { Asturiana }\end{array}$ & http://bit.ly/cla-fb & http://bit.ly/cla-yt & http://bit.ly/cla-ig \\
\hline $\begin{array}{l}\text { Corp. Alim. Peñasanta } \\
\text { (CAPSA Food) }\end{array}$ & Lácteo & Larsa & http://bit.ly/larsa-fb & http://bit.ly/larsa-yt & http://bit.ly/larsa-ig \\
\hline Danone & Lácteo & Danone & http://bit.ly/danone-fb & http://bit.ly/danone-yt & http://bit.ly/danone-ig \\
\hline Elpozo Alimentación & Cárnico & Elpozo & http://bit.ly/elpozo-fb & http://bit.ly/elpozo-yt & http://bit.ly/elpozo-ig \\
\hline Frigorifics Costa Brava & Cárnico & Costa Brava Foods & & & \\
\hline Grupo Comapa 2001 & Cárnico & Comapa & http://bit.ly/comapa-fb & & \\
\hline Grupo Jorge & Cárnico & $\begin{array}{l}\text { Campodulce } \\
\text { curados }\end{array}$ & http://bit.ly/campodulce-fb & & \\
\hline Grupo Lactalis Iberia & Lácteo & Lauki & http://bit.ly/lauki-fb & & \\
\hline Grupo Lactalis Iberia & Lácteo & Président & http://bit.ly/president-fb & & http://bit.ly/president-ig \\
\hline Grupo Lactalis Iberia & Lácteo & Puleva & http://bit.ly/puleva-fb & http://bit.ly/puleva-yt & http://bit.ly/puleva-ig \\
\hline $\begin{array}{l}\text { Industrias Cárnicas } \\
\text { Loriente Piqueras }\end{array}$ & Cárnico & Incarlopsa & http://bit.ly/incarlopsa-fb & & \\
\hline $\begin{array}{l}\text { Industrias Lácteas } \\
\text { Asturianas (ILAS) }\end{array}$ & Lácteo & Reny Picot & http://bit.ly/renypicot-fb & http://bit.ly/renypicot-yt & http://bit.ly/renypicot-ig \\
\hline Lácteas García Baquero & Lácteo & García Baquero & http://bit.ly/qgb-fb & http://bit.ly/qgb-yt & http://bit.ly/qgb-ig \\
\hline Mondelez España & Lácteo & Milka & http://bit.ly/milka-fb & http://bit.ly/milka-yt & http://bit.ly/milka-ig \\
\hline
\end{tabular}

Fuente: elaboración propia a partir de las redes sociales

Las 20 marcas analizadas suman más de 11,1 millones de seguidores en redes sociales, con una media de 558.971 seguidores por marca. Milka acumula el 84,3\% de los seguidores y el 96,7\% de los seguidores procede de Facebook. En el periodo analizado -año 2019 y primer semestre de 2020- se han monitorizado 5.904 publicaciones, repartidas como muestra la tabla 3: el 54,4\% en 
Facebook ( $n=3.210)$, el 8,2\% en YouTube $(n=482)$ y el 37,5\% en Instagram $(n=2.212)$. A excepción de Coren Grill, -el 86\% de sus publicaciones son en Instagram- Danone -con el 39,5\% de sus publicaciones en YouTube- y Milka -sin publicaciones en Facebook-, todas las marcas publican con mayor frecuencia en Facebook. Central Lechera Asturiana (n=771; 13,1\%) y Casa Tarradellas $(n=607 ; 10,3 \%)$ son las marcas con mayor número de publicaciones. La media de publicaciones mensuales para la muestra es de 328: 16,4 publicaciones en las tres redes sociales para cada marca y 5,5 publicaciones por red social y por marca.

Tabla 3. Publicaciones monitorizadas en el periodo de estudio

\begin{tabular}{|c|c|c|c|c|c|c|c|c|c|c|c|}
\hline \multirow{3}{*}{$\begin{array}{l}\text { Marca } \\
\text { Pascual }\end{array}$} & \multicolumn{3}{|c|}{ Facebook } & \multicolumn{3}{|c|}{ YouTube } & \multicolumn{3}{|c|}{ Instagram } & \multicolumn{2}{|c|}{ Total publicaciones } \\
\hline & \multirow{2}{*}{$\begin{array}{c}\text { Seguidores } \\
9.054\end{array}$} & \multicolumn{2}{|c|}{ Publicaciones } & \multirow{2}{*}{$\begin{array}{c}\text { Seguidores } \\
596\end{array}$} & \multicolumn{2}{|c|}{ Publicaciones } & \multirow{2}{*}{$\begin{array}{c}\text { Seguidores } \\
2.981\end{array}$} & \multicolumn{2}{|c|}{ Publicaciones } & \multirow{2}{*}{$\begin{array}{l}\text { Abs. } \\
580\end{array}$} & \multirow{2}{*}{$\begin{array}{c}\% \\
9,8 \%\end{array}$} \\
\hline & & 397 & $68,4 \%$ & & 39 & $6,7 \%$ & & 144 & $24,8 \%$ & & \\
\hline Campofrío & 126.867 & 212 & $57,5 \%$ & 14.400 & 40 & $10,8 \%$ & 5.305 & 117 & $31,7 \%$ & 369 & $6,3 \%$ \\
\hline Casa Tarradellas & 57.268 & 303 & $49,9 \%$ & 28.500 & 47 & $7,7 \%$ & 17.100 & 257 & $42,3 \%$ & 607 & $10,3 \%$ \\
\hline Lácteos COVAP & 3.473 & 191 & $48,8 \%$ & 600 & 70 & $17,9 \%$ & 2.795 & 130 & $33,2 \%$ & 391 & $6,6 \%$ \\
\hline Ibéricos COVAP & 9.788 & 256 & $67,9 \%$ & 91 & 9 & $2,4 \%$ & 7.025 & 112 & $29,7 \%$ & 377 & $6,4 \%$ \\
\hline Coren Grill & 300 & 1 & $1,8 \%$ & 462 & 7 & $12,3 \%$ & 1.510 & 49 & $86,0 \%$ & 57 & $1,0 \%$ \\
\hline Bonàrea Agrupa & 3.047 & 100 & $49,0 \%$ & 210 & 22 & $10,8 \%$ & 3.513 & 82 & $40,2 \%$ & 204 & $3,5 \%$ \\
\hline Central Lechera Asturiana & 181.318 & 401 & $52,0 \%$ & 4.580 & 42 & $5,4 \%$ & 36.000 & 328 & $42,5 \%$ & 771 & $13,1 \%$ \\
\hline Larsa & 11.014 & 164 & $46,5 \%$ & 222 & 26 & $7,4 \%$ & 1.777 & 163 & $46,2 \%$ & 353 & $6,0 \%$ \\
\hline Danone & 852.772 & 72 & $35,1 \%$ & 29.700 & 81 & $39,5 \%$ & 19.500 & 52 & $25,4 \%$ & 205 & $3,5 \%$ \\
\hline Elpozo & 74.130 & 270 & $48,9 \%$ & 2.060 & 61 & $11,1 \%$ & 8.515 & 221 & $40,0 \%$ & 552 & $9,3 \%$ \\
\hline Comapa & 1.153 & 75 & $100 \%$ & & & & & & & 75 & $1,3 \%$ \\
\hline Campodulce curados & 230 & 2 & $100 \%$ & & & & & & & 2 & $0,0 \%$ \\
\hline Lauki & 1.339 & 10 & $100 \%$ & & & & & & & 10 & $0,2 \%$ \\
\hline Président & 2.756 & 46 & $50,0 \%$ & & & & 978 & 46 & $50,0 \%$ & 92 & $1,6 \%$ \\
\hline Puleva & 153.249 & 225 & $75,0 \%$ & 12.600 & 9 & $3,0 \%$ & 3.869 & 66 & $22,0 \%$ & 300 & $5,1 \%$ \\
\hline Incarlopsa & 2.747 & 0 & & & & & & & & 0 & $0,0 \%$ \\
\hline Reny Picot & 9.184 & 236 & $51,2 \%$ & 8 & 8 & $1,7 \%$ & 920 & 217 & $47,1 \%$ & 461 & $7,8 \%$ \\
\hline García Baquero & 44.978 & 249 & $53,8 \%$ & 147 & 9 & $1,9 \%$ & 4.480 & 205 & $44,3 \%$ & 463 & $7,8 \%$ \\
\hline Milka & 9.267 .000 & 0 & $0,0 \%$ & 4.310 & 12 & $34,3 \%$ & 153.000 & 23 & $65,7 \%$ & 35 & $0,6 \%$ \\
\hline TOTAL & 10.811 .667 & 3.210 & $54,4 \%$ & 98.486 & 482 & $8,2 \%$ & 269.268 & 2.212 & $37,5 \%$ & 5.904 & $100,0 \%$ \\
\hline
\end{tabular}

Fuente: elaboración propia a partir de las redes sociales 
La base de los datos recopilados en la realización de la investigación puede consultarse en Castelló-Martínez (2020a). En Castelló-Martínez (2020b) pueden verse las capturas de pantalla de todas las publicaciones sobre bienestar animal identificadas.

\section{Resultados}

\subsection{Certificación y publicación en página web}

RQ1. ¿Las principales empresas de los sectores cárnico y lácteo cuentan con la certificación de bienestar animal? ¿Se informa en la web de las marcas de dicha certificación?

La mayoría de las principales marcas de los subsectores cárnico y lácteo cuentan con el sello de bienestar animal (n=19; 90,5\%) y así lo comunican en sus páginas web $(\mathrm{n}=16 ; 76,2 \%)$, incluso dedicando más de una sección a esta temática (n=8; 38,1\%), como muestra la figura 4. Esta información suele ubicarse en los apartados dedicados a la empresa -“quiénes somos" o "nuestras granjas" - o a la responsabilidad social corporativa, como en los casos de Larsa y ElPozo. En las secciones noticias o blog las marcas también destacan la certificación.

Figura 4. Información sobre bienestar animal en la página web

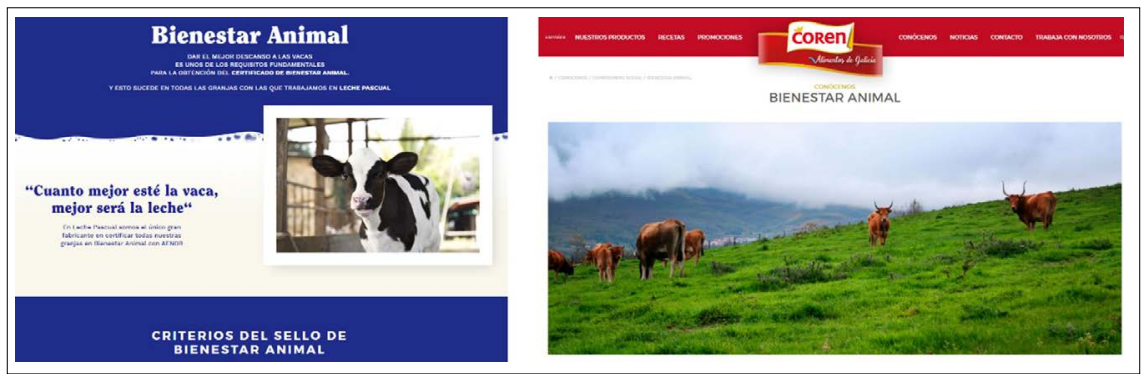

Fuente: https://lechepascual.es y https://coren.es

\subsection{Difusión en redes sociales}

RQ2. ¿Se realizan en redes sociales publicaciones relacionadas con el sello de bienestar animal? ¿Qué porcentaje representan estas publicaciones con respecto al total de publicaciones realizadas?

De las 20 marcas con presencia en redes sociales, 6 no realizan publicaciones relacionadas con esta cuestión (25\%): Campofrío, Campodulce Curados, Lauki, Président, Incarlopsa y Reny Picot. Es destacable el caso de Président, del Grupo Lactalis Iberia, ya que mientras Lauki y Puleva, pertenecientes al mismo grupo, sí publican contenidos relacionados con el bienestar animal, esta marca no lo hace. A pesar de contar con la certificación, comunicarlo en la página web y realizar publicaciones frecuentes en las tres redes sociales $(\mathrm{n}=369)$, Campofrío tampoco dedica contenidos en Facebook, YouTube o Instagram a este tema. 
Se identifica el recurso al bienestar animal en el 5,1\% de las publicaciones del periodo monitorizado ( $\mathrm{n}=299$ ): el 41,8\% en Facebook ( $n=125)$, el 19,7\% en YouTube $(n=59)$ y el 38,5\% en Instagram ( $n=115)$. YouTube es la red social en la que más publicaciones con este argumento se realizan con respecto a las publicaciones totales, superando el 12\%. Por anunciantes, Coren Grill, Central Lechera Asturiana y Larsa son las marcas que más publicaciones dedican al bienestar animal con respecto a las publicaciones totales: 15,8\%, 14,3\% y 10,5\%, respectivamente. El 36,8\% de las publicaciones identificadas pertenecen a Central Lechera Asturiana $(\mathrm{n}=110)$. Le siguen Larsa $(\mathrm{n}=37 ; 12,4 \%)$, Lácteos COVAP $(\mathrm{n}=35 ; 11,7 \%)$ y Pascual $(\mathrm{n}=32 ; 10,7 \%)$.

Por redes sociales y anunciantes, destacan los casos de Central Lechera Asturiana, Lácteos COVAP, Pascual y Larsa. La primera suma 62 publicaciones en Instagram, cifra que representa el 18,9\% de las publicaciones de la marca en esta red, el 56,4\% de las publicaciones de la marca sobre bienestar animal en redes sociales y el 53,9\% de las publicaciones sobre bienestar animal identificadas en Instagram. Esta misma marca suma 42 publicaciones relacionadas con esta cuestión en Facebook -el 33,6\% de las publicaciones sobre bienestar animal monitorizadas en esta plataforma-.

Lácteos COVAP es la marca con más vídeos sobre esta temática en YouTube ( $n=24)$, dato que suma el 34,3\% de las publicaciones de la marca en esta red, el 68,6\% de las publicaciones de la marca sobre bienestar animal en redes sociales y el 40,7\% de las publicaciones en YouTube sobre bienestar animal del total de la muestra. Pascual y Larsa hacen lo propio en Facebook, con 23 y 20 publicaciones, respectivamente -el 71,9\% y el 54,1\% de las publicaciones de cada marca en redes sociales sobre bienestar animal-.

\subsection{Audiencia e interacción}

RQ3. ¿Qué audiencia e interacción reciben las publicaciones en redes sociales de marcas de los sectores cárnico y lácteo sobre el sello de bienestar animal?

Con respecto a la audiencia y a la interacción de las publicaciones en redes sociales de los sectores cárnico y lácteo sobre el bienestar animal, los 299 mensajes suman más de 7 millones de visualizaciones y 185.623 interacciones, distribuidas de la siguiente manera: $98,4 \%$ me gusta, $0,1 \%$ no me gusta y $1,5 \%$ comentarios. Las publicaciones sobre bienestar animal reciben más me gusta en Instagram -el 68,9\% de los me gusta proceden de esta red- y más comentarios en Facebook, con el 63,8\% de los comentarios totales.

Los mensajes de Central Lechera Asturiana alcanzan el 93,2\% de los me gusta y el 80,2\% de los comentarios. Son también de esta marca las publicaciones sobre bienestar animal que reciben más interacción, con seis publicaciones que superan los 5.000 me gusta y los 70 comentarios cada una de ellas. Las cinco publicaciones en Instagram con más interacción de Central Lechera Asturiana -y del total de la muestra- son de un concurso que la marca lanzó entre los seguidores, bajo la etiqueta \#MiPradoEsTuCasa. En la figura 5 se muestran dos de las publicaciones de esta marca más populares: 
Figura 5. Publicaciones de Central Lechera Asturiana

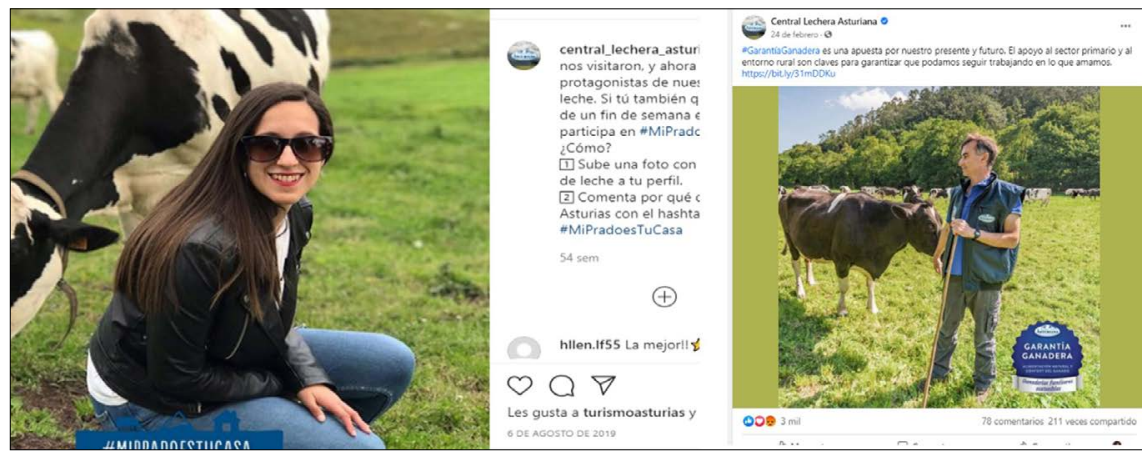

Fuente: Instagram (http://bit.ly/ba-cla-196) y Facebook (http://bit.ly/ba-cla-147)

Si comparamos la interacción -en términos de me gusta y comentarios por ser las variables presentes en las tres redes socialesde las 5.904 publicaciones monitorizadas con la de las 299 publicaciones relacionadas con el bienestar animal, observamos que la media de me gusta y comentarios es más elevada en estas últimas: 611 me gusta y 9,6 comentarios por publicación relacionada con el bienestar animal frente a 563,2 me gusta y 7,8 comentarios por publicación.

\subsection{Estrategia creativa}

RQ4. ¿Existen características comunes en las publicaciones sobre el sello de bienestar animal de las marcas de los sectores cárnico y lácteo en redes sociales?

En cuanto a las características formales de los contenidos en redes sociales sobre bienestar animal, el $64,5 \%$ de las publicaciones se realizan en sábado ( $n=193$ ). No existe ninguna publicación únicamente con texto; de hecho, el 98,3\% de los mensajes combinan el texto con algún otro tipo de contenido ( $\mathrm{n}=294)$. La mayoría de las publicaciones incluyen etiquetas ( $\mathrm{n}=232 ; 77,6 \%)$, como las siguientes: \#BienestarAnimalPascual (Pascual), \#BienestarAnimal (Casa Tarradellas), \#BienestarAnimalbonArea (BonÀrea), \#GarantíaGanadera, \#MiPradoEsTuCasa, \#LoNaturalSabeMejor (Central Lechera Asturiana), \#LeiteDePastoreo, \#PastoreoLarsa, \#VacasFelices (Larsa) o \#LoBuenoUne (García Baquero).

El tipo de contenido más habitual publicado por las marcas de los subsectores cárnico y lácteo en redes sociales sobre bienestar animal son las imágenes ( $\mathrm{n}=122 ; 40,8 \%$ ), los vídeos ( $\mathrm{n}=88 ; 29,4 \%)$ y los microvídeos de menos de 20 segundos ( $\mathrm{n}=42 ; 14 \%)$. De acuerdo con la clasificación de Costa-Sánchez (2017), los vídeos más comunes son los spots cortos -menos de 1 minuto de duración- ( $\mathrm{n}=27 ; 30,6 \%)$, los vídeos corporativos ( $\mathrm{n}=17 ; 19,3 \%)$ y los de responsabilidad social empresarial ( $\mathrm{n}=17 ; 19,3 \%)$.

Los enlaces a contenidos relacionados $(n=114 ; 38,1 \%)$ y las llamadas a la acción $(n=113 ; 37,8 \%)$ no son frecuentes. Las marcas suelen animar a seguir a la empresa en redes sociales $(n=49)$ o lanzar preguntas a seguidores $(n=44)$ cuando publican mensajes sobre bienestar animal. La relación de este tipo de mensajes con algún tipo de incentivo promocional -concursos, promociones, sorteos- tampoco es habitual: encontramos 28 publicaciones con concursos -el 89,3\% son de Central Lechera Asturiana, con 
un concurso en el que los usuarios se convierten en los protagonistas-. En la figura 6 se muestra un ejemplo de publicación con concurso de Pascual:

Figura 6. Publicación de Pascual sobre bienestar animal con concurso

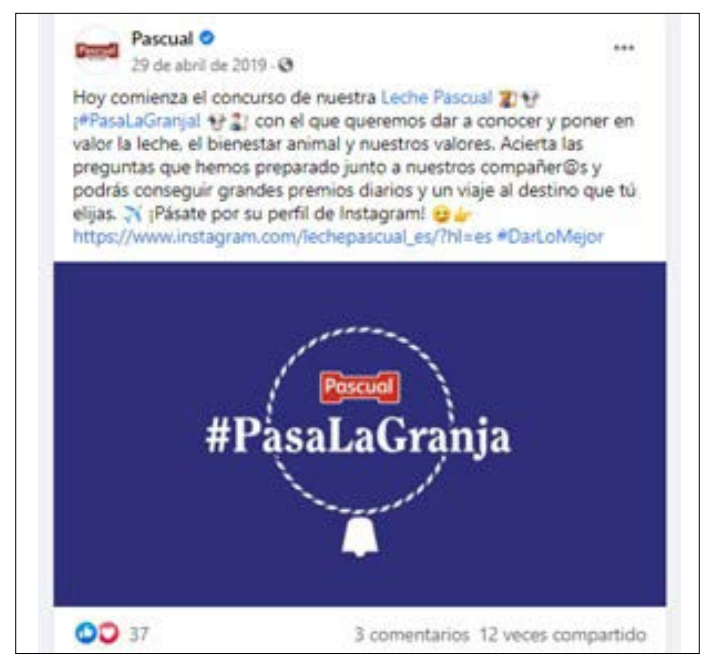

Fuente: Facebook (http://bit.ly/ba-pascual-11)

La asociación de los contenidos sobre bienestar animal con mensajes de tipo corporativo, como aquellos relacionados con la responsabilidad social empresarial, o con la comunicación persuasiva de la marca -en el caso de los spots-, y la escasa existencia de incentivos promocionales en este tipo de publicaciones indican el valor estratégico en el posicionamiento diferencial de la comunicación de marca que tiene la cuestión del bienestar animal.

Por último, como rasgos comunes a la mayoría de publicaciones analizadas sobre bienestar animal en redes sociales destacan el tono emocional del mensaje -apelando al amor por lo natural, al cariño y a la pasión, como hace Larsa al hablar de vacas felices- y el compromiso con el cuidado animal como un valor tradicional y un legado familiar-Pascual habla de "una filosofía que nos acompaña desde siempre", Lácteos COVAP destaca que "el tiempo ha unido a generaciones de hombres y mujeres honestos y comprometidos con un modo de vida entre la tradición y la innovación” y Central Lechera Asturiana afirma que "la ganadería es para nosotros mucho más que nuestra forma de vida: es la herencia de nuestros padres, que supieron transmitirnos el buen hacer y el amor por lo que hacemos"-.

El compromiso con la sostenibilidad, las mejoras en las instalaciones y el vínculo con la tierra -especialmente en el caso de Central Lechera Asturiana- son argumentos que acompañan a la comunicación del bienestar animal. Las imágenes más frecuentes muestran a las vacas pastando en libertad y a los ganaderos cuidándolas, en el caso del subsector lácteo, y el producto final en el subsector cárnico, como muestra la figura 7 : 
Figura 7. Publicaciones sobre bienestar animal de Larsa, Casa Tarradellas y García Baquero

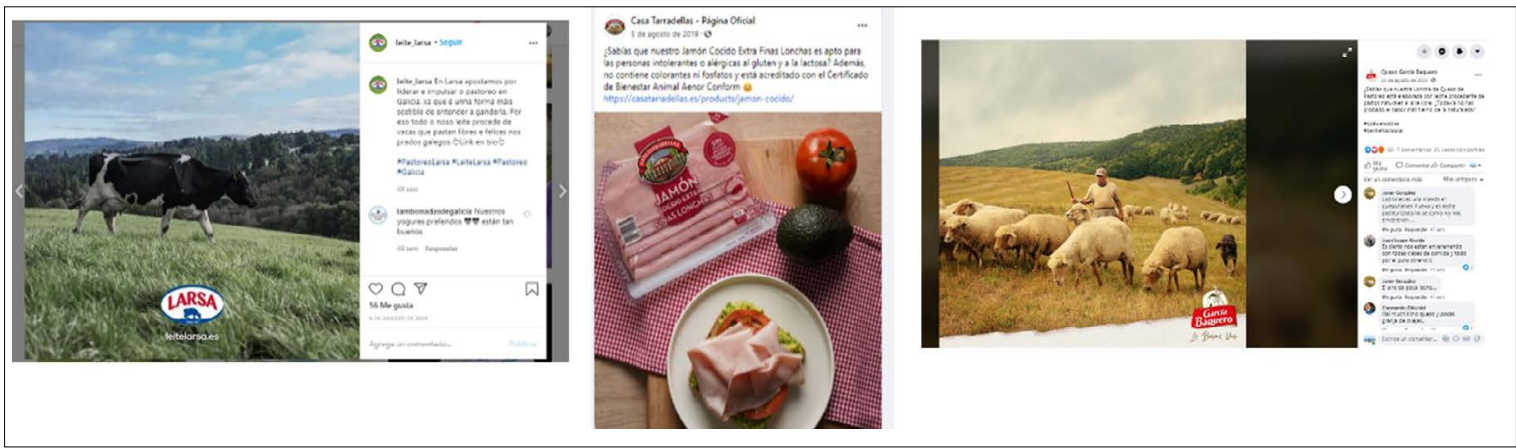

Fuente: Instagram (http://bit.ly/ba-larsa-260 y Facebook (http://bit.ly/ba-casatarradellas-33 y http://bit.ly/ba-qgb-291)

\section{Discusión y conclusiones}

Los resultados avalan la presencia del bienestar animal como eje de comunicación en las estrategias creativas de la comunicación digital de las marcas de los sectores cárnico y lácteo. Frente a argumentos tradicionalmente presentes en la publicidad de alimentos como la salud, la variedad o el envase (Díaz, 2003; Rey, 2012), la presencia del bienestar animal como eje de la comunicación persuasiva en entornos digitales de las marcas estudiadas demuestra que la ecología, como facilitadora de la calidad y la salud alimentarias, gana peso como ventaja diferencial.

Los contenidos que las marcas de alimentación difunden en redes sociales se presentan como mensajes híbridos (Balasubramanian, 1994) en los que la intencionalidad comercial se fusiona con contenidos, en este caso especialmente informativos, que pretenden transmitir el compromiso de la marca con el cuidado de los animales y una alimentación sana, conectando de esta manera con una preocupación del consumidor. Estos mensajes fomentan la interacción y la participación del usuario, característica intrínseca a la comunicación digital gracias a la cultura colaborativa y la sociedad participativa que instauró la democratización tecnológica (Castelló-Martínez y Del Pino-Romero, 2019).

A la calidad y la seguridad alimentarias y el aporte nutricional beneficioso para la salud se suma hoy la preocupación del consumidor por la correcta gestión en los sistemas de cría de animales destinados a la producción alimentaria, un reto al que debe dar respuesta el sector alimentación. El bienestar animal cuenta con una legislación cada vez más exigente y las buenas prácticas al respecto tienen un impacto directo en la calidad del producto y en la reducción de costes, con modelos de producción más eficientes y sostenibles. Son necesarios, en este sentido, un compromiso franco y duradero y una comunicación coherente, transparente y honesta.

El histórico de crisis reputacionales vividas por el sector alimentación (Saura, 2005) constata la importancia que adquiere en este sector una gestión profesionalizada de la comunicación ante la sensibilidad de la población a la seguridad alimentaria. Además, son numerosos los estudios que han demostrado la relación causal de la publicidad alimentaria con una dieta poco saludable y 
la obesidad, especialmente entre públicos más sensibles como el infantil (Chou, Rashad y Grossman, 2008; Veerman et al, 2009; Chapman et al, 2012; Moreno y Luque, 2014).

Con iniciativas empresariales y soluciones comunicativas que dan respuesta a tensiones sociales y culturales del contexto como el bienestar animal y el fomento de una alimentación sana, las empresas ansían alcanzar el estadio de marcas útiles, marcas que quieren contribuir a construir un mundo mejor. Pese a todo, diversos estudios evidencian que a las empresas todavía les queda mucho por hacer en el uso de los medios sociales en aras de la transparencia, la conversación y la coherencia entre el decir y el hacer (Gómez, 2013; Aced y Lalueza, 2016; Zeler y Capriotti, 2019).

Las limitaciones del estudio vienen dadas por el tamaño muestral y la escasa literatura científica previa sobre el tema de estudio. Futuras líneas de investigación plantean ampliar el estudio a otros subsectores de alimentación, como el de la pesca, así como analizar la evolución temporal del recurso al bienestar animal en las estrategias creativas en comunicación digital.

\section{Agradecimientos}

Este artículo ha sido traducido al inglés por Brian O’Halloran.

\section{Referencias bibliográficas}

Aced Toledano, C.; Lalueza Bosch, F. (2016). ¿Qué contenidos publican las empresas en los medios sociales? Análisis crítico del discurso de las compañías del IBEX 35 y del Fortune 500 en blogs corporativos, Facebook y Twitter. Revista internacional de relaciones públicas, 6(11), 135-154. https://doi.org/10.5783/RIRP-11-2016-08-135-154

AECOC (2020). Los indicadores de compra y consumo de productos cárnicos 2019. Recuperado de http://bit.ly/aecoc-2020

Alonso, M.E.; González-Montaña, J.R.; Lomillos, J.M. (2020). Consumers' Concerns and Perceptions of Farm Animal. Welfare. Animals, 10, 385. https://doi.org/10.3390/ani10030385

Balasubramanian, S. K. (1994). Beyond Advertising and Publicity: Hybrid Messages and Public Policy Issues. Journal of Advertising, 23(4), 29-46. https://doi.org/10.1080/00913367.1943.10673457

Braunsberger, K. (2015). The Impact of Animal Welfare Advertising on Opposition to the Canadian Seal Hunt and Willingness to Boycott the Canadian Seafood Industry. Anthrozoös. A multidisciplinary journal of the interactions of people and animals, 27(1), 111-125. https://doi.org/10.2752/175303714X13837396326530

Business Benchmark on Farm Animal Welfare (BBFAW) (2020). The Business Benchmark on Farm Animal Welfare 2019. Recuperado de http://bit.ly/bbfaw-2020

Barlovento Comunicación (2019). Análisis mensual del comportamiento de la audiencia TV. Abril 2021. Recuperado de http:// bit.ly/barlovento-abril-2021

Castelló-Martínez, A. (2020a). Base de datos del estudio sobre la comunicación del bienestar animal en redes sociales. Recuperado de http://bit.ly/ba-basededatos 
Castelló-Martínez, A. (2020b). Publicaciones sobre bienestar animal en redes sociales de las empresas de los sectores cárnico y lácteo. Recuperado de http://bit.ly/ba-capturas

Castelló-Martínez, A.; Del Pino-Romero, C. (2019). De la publicidad a la comunicación persuasiva integrada. Estrataegia y empatía. Madrid: Esic.

Chapman, C.D.; Benedict, C.; Brooks, S.J.; Schlöth, H. B. (2012). Lifestyle determinants of the drive to eat: A meta-analysis. The American Journal of Clinical Nutrition, 96, 492-497. https://doi.org/10.3945/ajcn.112.039750

Chou, S. Y.; Rashad, I.; Grossman, M. (2008). Fast-food restaurant advertising on television and its influence on childhood obesity. The Journal of Law and Economics, 51(4), 599-618. https://doi.org/10.1086/590132

Comisión Europea (2019). Online consultation on the Future of Europe Second interim report. Recuperado de http://bit.ly/ ce-ba-2019

Comisión Europea (2015). Attitudes of Europeans towards Animal Welfare. Recuperado de http://bit.ly/ce-ba-2015

Costa-Sánchez, C. (2017). Estrategias de videomarketing online: tipología por sectores de negocio. Communication \& Society, 30(1), 17-38. Recuperado de http://bit.ly/costasanchez-2017

Díaz Rojo, J. A. (2003). Lenguaje y reclamos de salud en la publicidad de los alimentos. Anàlisi, 30, 217-224. Recuperado de http:// bit.ly/diaz-2003

Díaz-Méndez, C.; González-Álvarez, M. (2013). La problematización de la alimentación: un recorrido sociológico por la publicidad alimentaria (1960-2010). EMPIRIA. Revista de Metodología de Ciencias Sociales, 25, 121-146. https://doi.org/10.5944/ empiria.25.2013.3800

El Mundo (2018). Lidl deja de vender huevos de gallinas enjauladas. 17/01/2018. Recuperado de http://bit.ly/elmundo-lidl-2018 Federación de Industrias de Alimentación y Bebidas (FIAB) (2020). Alimentación en España 2020. Recuperado de http://bit.ly/ fiab-2020

Gómez Vásquez, L. M. (2013). Me gusta o te sigo: Análisis de la comunicación de prácticas de Responsabilidad Social Corporativa a través de los medios sociales. Correspondencias \& análisis, 3, 89-109. https://doi.org/10.24265/cian.2013.n3.04

Hewson, C. J. (2005). Why the Theme Animal Welfare? Journal of Veterinary Medical Education, 32(4), 416-418. https://doi. org/10.3138/jvme.32.4.416

IAB Spain (2021). Estudio de Redes Sociales 2021. Recuperado de http://bit.ly/iab-redes-2021

IAB Spain (2020). Observatorio de Marcas en Redes Sociales 2020. Recuperado de http://bit.ly/iab-observatorio-2020 InfoAdex (2021). Estudio InfoAdex de la Inversión Publicitaria en España 2021. Recuperado de http://bit.ly/infoadex-2021

Kantar Worldpanel (2020). Brand Footprint España 2020. Recuperado de http://bit.ly/kantar-2020

López-Briones Reverte, C. (2017). Construcción del concepto de alimentación saludable a través de la publicidad, España 19602013. Tesis Doctoral. Alicante: Universidad de Alicante. Recuperado de http://bit.ly/lopez-briones-2017 
Ministerio de Agricultura, Pesca y Alimentación (2020). Informe de Consumo Alimentario en España 2019. Recuperado de http:// bit.ly/mapa-2020

Moreno, M.; Luque, E. (2014). Comer por los ojos: la publicidad alimentaria y sus riesgos. Panorama Social, 19, 49-62. Recuperado de http://bit.ly/moreno-luque-2014

Raya González, P. (2017). Estrategias comunicativas de la publicidad de Campofrío en Twitter. Tesis Doctoral. Granada: Universidad de Granada. Recuperado de http://bit.ly/raya-tesis-2017

Rey, J. (2012). Publicidad y cambio alimentario. Estudio de las razones de compra utilizadas en la publicidad de alimentos española. Doxa Comunicación, 15, 127-150. Recuperado de http://bit.ly/rey-2012

Rey Fuente, J. (2010). Publicidad de productos de alimentación y productos vigoréxicos. ¿Una cuestión de límite? Icono 14 Revista Científica De Comunicación Y Tecnologías Emergentes, 8(3), 143-169. https://doi.org/10.7195/ri14.v8i3.233

Saura, P. (2005). Las crisis generales y particulares sufridas por el sector de alimentación en España, sus causas y su gestión de comunicación de crisis. En Rodríguez Patrón, A. (Coord.). Tendencias actuales en las relaciones públicas. II Congreso Internacional de Investigadores en Relaciones Públicas (223-236). Sevilla: Asociación de Investigadores en Relaciones Públicas (AIRP). Recuperado de http://bit.ly/saura-2005

Sullivan, M.; Longnecker, N. (2010). Choosing Effective Frames to Communicate Animal Welfare Issues. 11th International Conference on Public Communication of Science and Technology (PCST) New Delhi, India. Recuperado de http://bit.ly/sullivan-2010

Valero, M. (2018). Bronca de los ganaderos con Pascual o Lidl por su leche de bienestar animal. El Confidencial, 30/09/2018. Recuperado de http://bit.ly/valero-confidencial-2018

Veerman, J.L.; Van Beeck, E.F.; Berendregt, J.J.; Mackenbach, J. P. (2009). By how much would limiting TV food advertising reduce childhood obesity? European Journal of Public Health, 19(4), 365-369. https://doi.org/10.1093/eurpub/ckp039

Vigors, B. (2019). Citizens' and Farmers' Framing of 'Positive Animal Welfare' and the Implications for Framing Positive Welfare in Communication. Animals, 9(4), 147. https://doi.org/10.3390/ani9040147

Youssef Elghannam, A. A. M. (2019). Las redes sociales como una nueva herramienta para el marketing en línea de los alimentos. Tesis Doctoral. Badajoz: Universidad de Extremadura. Recuperado de http://bit.ly/youssef-2019

Zeler, I.; Capriotti, P. (2019). Communicating corporate social responsibility issues on Facebook's corporate fan pages of latin American companies. El profesional de la información, 28(5), e280507. https://doi.org/10.3145/epi.2019.sep.07 